\title{
The density evolution of the killed Mckean-Vlasov process
}

\author{
Peter E. Caines * Daniel Ho, ${ }^{\dagger} \quad$ Qingshuo Song, ${ }^{\ddagger} \quad \S$
}

\begin{abstract}
The study of the density evolution naturally arises in Mean Field Game theory for the estimation of the density of the large population dynamics. In this paper, we study the density evolution of McKean-Vlasov stochastic differential equations in the presence of an absorbing boundary, where the solution to such equations corresponds to the dynamics of partially killed large populations. By using a fixed point theorem, we show that the density evolution is characterized as the unique solution of an integro-differential Fokker-Planck equation with Cauchy-Dirichlet data.
\end{abstract}

\section{Introduction}

Let $W$ be a $\mathbb{R}^{d}$-valued Brownian motion on a filtered probability space $\left(\mathbb{P}, \Omega, \mathcal{F},\left\{\mathcal{F}_{t}\right\}_{t \geq 0}\right)$, and we consider the stochastic differential equation

$$
d X_{t}=\bar{b}\left(X_{t}, t\right) d t+d W_{t} ; X_{0} \sim m_{0},
$$

where $X_{0}$ is the given initial state with its probability density $m_{0}$ on $\mathbb{R}^{d}$. A well known result, see for instance Hormander's Theorem in Section V.38 of [19], says that the density $m(x, t)=\mathbb{P}\left(X_{t} \in\right.$ $d x) / d x$ satisfies Fokker-Planck equation (FPK) with Cauchy data

$$
\begin{cases}\partial_{t} m=-\operatorname{div}_{x}(\bar{b} m)+\frac{1}{2} \Delta m, & (0, \infty) \times \mathbb{R}^{d} \\ m(x, 0)=m_{0}(x) & x \in \mathbb{R}^{d}\end{cases}
$$

Recently, Mean Field Game theory attracted a great deal of attention in the control and other fields after it was initiated in a series of founding works by Huang, Caines, and Malhame (e.g., $[13,14,15])$, and independently in that of Lasry and Lions (e.g., [17]). This led to extensive studies on the density evolution of McKean-Vlasov type stochastic differential equations (MV-SDE) of the following form:

$$
d X_{t}=b\left(X_{t}, \mathbb{E}\left[X_{t}^{p}\right]\right) d t+d W_{t} ; \quad X_{0} \sim m_{0}
$$

for some positive integer $p$, see, for instance, $[2,4,12]$ and the references therein. It is well known that the density follows the integro-differential FPK

$$
\begin{cases}\partial_{t} m=-\operatorname{div}_{x}\left(b\left(x, \int_{\mathbb{R}^{d}} x^{p} m(x, t) d x\right) m\right)+\frac{1}{2} \Delta m, & (0, \infty) \times \mathbb{R}^{d}, \\ m(x, 0)=m_{0}(x), & x \in \mathbb{R}^{d} .\end{cases}
$$

\footnotetext{
*Department of Electrical and Computer Engineering, McGill University, Montreal, Canada. peterc@cim.mcgill.ca $\dagger$ Department of Mathematics, City University of Hong Kong, Hong Kong. madaniel@cityu.edu.hk.

${ }^{\ddagger}$ Department of Mathematics, Worcester Polytechnic University, and Department of Mathematics, City University of Hong Kong, Hong Kong. qsong@wpi.edu.

$\S$ This research has been partially supported by GRF CityU 11201518.
} 
In this paper, we study a similar integro-differential FPK associated to population density dynamic of the process (1) killed at the boundary of the unit ball. Indeed, the setup of killed population has been already applied to mean field games in different contexts recently, see for instance, [7], [5], [9], and [3]. One of the closest references to our setup might be [3], where FPK similar to our current study has been briefly sketched, see Page 2217 [3]. However, the corresponding study on the solvability and regularities of FPK for the killed population is not available to the best of our knowledge.

It is noted that, due to the loss of population at the boundary, the killed process is a strictly submarkovian in $B_{1}$. As a result, its associated FPK is given with Dirichlet data along the boundary in addition to the counter-part FPK of unkilled process (2). Our goal is to legitimate the following statement: Under appropriate conditions on the drift function $b(\cdot, \cdot)$, the density of killed process is the classical solution of its associated Integro-Differential FPK with initial-boundary data.

In this paper, we first present the precise problem formulation and its main result on the characterization of the density in Section 2. In Section 3, we present the detailed proof, which is mainly based on the argument of the Leray-Schauder fixed point theorem. Section 4 is the summary, and the last section is Appendix for some useful facts collected from existing literatures.

\section{Motivation, problem setup and main results}

\subsection{Motivation}

The role of FPK equation in Mean Field Game theory can be illustrated via the following scenario.

- Given a particle system of population size $N$, our interest is the evolution of the mean field term given by

$$
Z_{t}^{N}:=\frac{1}{N} \sum_{i=1}^{N}\left(X_{t}^{i, N}\right)^{p}
$$

In the above, we only consider $p$ as a given positive integer. If $p=1$, then the mean field term $Z_{t}^{N}$ may refer to the population $p$ th mean. Suppose the position $X_{t}^{i, N}$ of the $i$ th particle follows the dynamics

$$
d X_{t}^{i, N}=b\left(X_{t}^{i, N}, Z_{t}^{N}\right) d t+d W_{t}^{i} ; \quad X_{0}^{i, N} \sim m_{0}, i=1,2, \ldots, N .
$$

driven by an independent Brownian motion $W^{i}$ with i.i.d. initial distribution $X_{0}^{i, N} \sim m_{0}$, then one could solve a system of $N$-equations of (4) to track the mean field term $Z_{t}^{N}$, which bears high computation cost as $N$ becomes large.

On the other hand, if $N$ is a large number, the law of large number implies that $Z_{t}^{N}$ can be effectively approximated by a deterministic process $\int_{\mathbb{R}^{d}} x^{p} m(x, t) d x$ for large number $N$ in the sense that

$$
\lim _{N \rightarrow \infty} Z_{t}^{N}=\int_{\mathbb{R}^{d}} x^{p} m(x, t) d x, \quad \text { almost surely } \forall t>0 .
$$

In the above, the function $m(\cdot, t)$ is the density of $X_{t}$ of (1) at its continuum limit, or equivalently is the solution of FPK (2). Indeed, according to the application of the Hewitt and Savage Theorem (see Theorem 5.14 of [4]), the FPK (2) can be used for a fairly broadly defined class of symmetric functionals in a large system at its continuum.

A similar argument may also be resorted to for a partially killed $N$-particle system: 
- Suppose the process $X^{i, N}$ of the $i$ th particle following MV-SDE (4) has an open unit ball $B_{1}$ as its state space. This means that $X^{i, N}$ explodes (absorbed) at the first exit time $\zeta^{i, N}$ from $B_{1}$. If we denote the population at time $t$ by

$$
L_{t}:=\sum_{i=1}^{N} I_{(t, \infty)}\left(\zeta^{i, N}\right),
$$

then the $L_{t}$ is monotonically decreasing from the initial size $L_{0}=N$ to 0 as $t$ goes to infinity. If we again consider the mean field term, then the law of large number implies that

$$
Y_{t}^{N}:=\frac{1}{N} \sum_{i=1}^{N}\left(X_{t}^{i, N}\right)^{p} I_{(t, \infty)}\left(\zeta^{i, N}\right) \rightarrow \int_{B_{1}} x^{p} m(x, t) d x, \quad \text { almost surely } \forall t>0 .
$$

In the above, $m(\cdot, t)$ is the density of killed generic process $X_{t}$ in $B_{1}$ with its absorbing boundary.

Therefore, a characterization of the density $m$ in terms of its associated FPK is desirable for the killed process, however it's not available in the literature to the best of authors' knowledge.

\subsection{Problem setup}

$W$ is a $\mathbb{R}^{d}$-valued Brownian motion on a filtered probability space $\left(\mathbb{P}, \Omega, \mathcal{F},\left\{\mathcal{F}_{t}\right\}_{t \geq 0}\right)$. Let $B_{1}$ be the open unit ball in $\mathbb{R}^{d}$ and $p$ be a positive integer. We consider MV-SDE of the form (1) with the only difference on the state space $B_{1}$. To emphasize its state space, we write the state space $B_{1}$ after semicolon together with given initial state $X_{0}$,

$$
d X_{t}=\left(b\left(X_{t}, \mathbb{E}\left[X_{t}^{p} ; B_{1}\right]\right) d t+d W_{t}\right) I_{B_{1}}\left(X_{t}\right) ; \quad X_{0} \sim m_{0} .
$$

In the above, the function $b: B_{1} \times B_{1} \ni(x, y) \mapsto b(x, y) \in \mathbb{R}^{d}$ is a given drift and $X_{0}: \Omega \mapsto B_{1}$ is a given $\mathcal{F}_{0}$-measurable its initial state with its density $m_{0}$ on $B_{1}$. Moreover, the mean field term in the drift function is understood as

$$
\mathbb{E}\left[X_{t}^{p} ; B_{1}\right]:=\mathbb{E}\left[X_{t}^{p} I_{B_{1}}\left(X_{t}\right)\right]=\int_{B_{1}} x^{p} m(x, t) d x .
$$

Note that, the boundary $\partial B_{1}$ is set to be the absorbing boundary, i.e. once $X_{t}$ reaches the cemetery $\partial B_{1}$, the term $I_{B_{1}}\left(X_{t}\right)$ leads to $d X_{t}=0$ afterwards and it never returns to $B_{1}$. As in the convention, we refer

$$
\zeta=\inf \left\{t>0: X_{t} \notin B_{1}\right\}
$$

as the lifetime of $X$. We are interested in

1. the existence and uniqueness of the solution of MV-SDE (6) up to the lifetime $\zeta$, and further

2. its density evolution $m(x, t)=\mathbb{P}\left(X_{t} \in d x\right) / d x$ for $(x, t) \in B_{1} \times \mathbb{R}^{+}$, if it exists.

The above questions are well studied for MV-SDE if the state $X_{t}$ is valued in the whole space $\mathbb{R}^{d}$. However, if the state space is the bounded set $B_{1}$, one shall note that $\mathbb{P}\left(X_{t} \in B_{1}\right)<1$ for $t>0$, hence the process $X_{t}$ has to be a submarkovian (see the definition of submarkvoian in Page 9 of [6]). Indeed, due to the positive probability of the explosion on any time interval $(0, t)$, one can obtain,

$$
\int_{B_{1}} m(x, t) d x=\mathbb{P}\left(X_{t} \in B_{1}\right)=1-\mathbb{P}\left(X_{t} \in \partial B_{1}\right)<1, \forall t>0,
$$

as long as the solution $X$ of (1) exists. To proceed, let us be precise with the definition of the solution of $(6)$. 
Definition 1 Given a filtered probability space $\left(\mathbb{P}, \Omega, \mathcal{F},\left\{\mathcal{F}_{t}\right\}_{t \geq 0}\right)$ with a $\mathbb{R}^{d}$-valued Brownian motion $W$, and a $B_{1}$-valued $\mathcal{F}_{0}$-measurable random variable $X_{0}$ for its initial state, a process $X$ is said to be the solution of (6) (up to its explosion) with its state space $B_{1}$, if $(X, \beta)$ satisfies both

$$
d X_{t}=\left(b\left(X_{t}, \beta_{t}\right) d t+d W_{t}\right) \cdot I_{B_{1}}\left(X_{t}\right), \quad X_{0} \sim m_{0},
$$

and

$$
\beta_{t}=\mathbb{E}\left[X_{t}^{p} I_{B_{1}}\left(X_{t}\right)\right]
$$

For a two-variable function $m: \mathbb{R}^{d} \times \mathbb{R} \ni(x, t) \mapsto m(x, t) \in \mathbb{R}$, we often treat $m_{t}(x)=m(x, t)$ as a function-valued evolution in time. If $\beta_{t}$ were given by a known deterministic process in (7) in priori, then one can directly write its density evolution as of FPK (20) with the substitution $\bar{b}(x, t)=b\left(x, \beta_{t}\right)$. At least heuristically, one can next use (8) to replace $\beta_{t}$ in FPK (20) by $\int_{B_{1}} x^{p} m_{t} d x$, and obtain a new FPK of the following form for its density evolution:

$$
\begin{cases}\partial_{t} m=\frac{1}{2} \Delta m-\operatorname{div}_{x}\left(m_{t}(x) b\left(x, \int_{B_{1}} x^{p} m_{t}(x) d x\right)\right), & \text { on } B_{1} \times(0, \infty) ; \\ m(x, 0)=m_{0}(x), & \text { on } \bar{B}_{1} ; \\ m(x, t)=0, & \text { on } \partial B_{1} \times(0, \infty) .\end{cases}
$$

In the above (9), the divergence term shall reads

$$
\operatorname{div}_{x}\left(m_{t}(x) b(x, y)\right)=\sum_{i=1}^{d} \partial_{x_{i}}\left(m_{t}(x) b^{i}(x, y)\right), \text { where } y=\int_{B_{1}} x^{p} m_{t}(x) d x .
$$

\subsection{Main result}

To present our main results, we shall impose the following regularity assumptions throughout the text:

(A1) $b \in C^{1+\gamma}\left(B_{1}^{2} ; \mathbb{R}^{d}\right)$ and $m_{0} \in C_{0}^{2+\gamma}\left(B_{1}\right)$ for some $\gamma \in(0,1]$.

In the above assumption, we adopt the notion $C^{k+\gamma}\left(B_{1}^{2} ; \mathbb{R}^{d}\right)$ from [16] to denote the collection of all functions $f: B_{1}^{2} \mapsto \mathbb{R}^{d}$ which has all of their $k$ th derivatives are $\gamma$-Hölder continuous. By $C_{0}^{k+\gamma}\left(B_{1}\right)$, we refer the space of functions $f: B_{1} \mapsto \mathbb{R}$ in $C^{k+\gamma}\left(B_{1}, \mathbb{R}\right)$ which is smoothly vanishing to zero outside of its domain, see more details about Hölder space in Definition 8 of Appendix.

Theorem 2 If we assume (A1), then there exists a solution of MV-SDE (6), whose density satisfies FPK (9).

\subsection{An example}

Next, we provide a special case of Theorem 2, where the statement of Theorem 2 could be easily verified from the symmetry of the initial density directly using Fourier series. We relegate the proof for the general case to Section 3.

We consider 1-d MV-SDE (6) with the drift and initial density given by

$$
b(x, y)=y^{2}, m_{0}(x)=\kappa e^{1 /\left(x^{2}-1\right)} I_{(-1,1)}(x),
$$

where $\kappa$ is the normalization constant

$$
\kappa=\left(\int_{-1}^{1} e^{1 /\left(x^{2}-1\right)} d x\right)^{-1} .
$$


It is noted that both $b$ and $m_{0}$ satisfy (A1). We use the following $L^{2}(-1,1)$-orthogonal basis: for all natural number $n$

$$
\eta_{n}(x)=\sin (n \pi(x+1) / 2), \forall x \in(-1,1) .
$$

By [20], the equation

$$
\begin{cases}\partial_{t} m=\frac{1}{2} \partial_{x x} m & \text { on }(-1,1) \times(0, \infty) \\ m(x, 0)=m_{0}(x), & \text { on }[-1,1] ; \\ m( \pm 1, t)=0, & \text { on }(0, \infty)\end{cases}
$$

has the unique solution

$$
m(x, t)=\sum_{n \in \mathbb{N}}\left(m_{0}, \eta_{n}\right) e^{-n^{2} \pi^{2} t / 8} \eta_{n} .
$$

By [8], the above function $m$ given by Fourier series is also the density function of a Brownian motion with initial distribution $m_{0}$ absorbed at $\{ \pm 1\}$, i.e.

$$
d X_{t}=d W_{t} \cdot I_{(-1,1)}\left(X_{t}\right) ; \quad X_{0} \sim m_{0} .
$$

If $n$ is even, then $\left(m_{0}, \eta_{n}\right)=0$, since $m_{0}$ is even and $\eta_{n}$ is odd. Therefore, $v$ is an even function of the form

$$
m(x, t)=\sum_{n=o d d}\left(m_{0}, \eta_{n}\right) e^{-n^{2} \pi^{2} t / 8} \eta_{n} .
$$

Hence, if $p$ is odd, we have

$$
\beta_{t}=\mathbb{E}\left[X_{t}^{p} I_{(-1,1)}\left(X_{t}\right)\right]=0, \forall t \geq 0 .
$$

Moreover, we observe that, due to the fact of $b\left(x, \beta_{t}\right)=b(x, 0)=0,(11)$ and (12) are equivalent to (9) and (6), respectively. Thus we conclude that

- With the setup (10), if $p$ is an odd number, then the density function of (6) has an explicit form (13), and solves (9).

We also observe that, the density $m(x, t)$ goes to zero function as $t \rightarrow 0$, and zero function is actually stationary distribution of such a process. It's not hard to see this example has the following extensions: If there exists some $\gamma \in(0,1]$ such that,

- $b \in C^{1+\gamma}\left(B_{1}^{2} ; \mathbb{R}^{d}\right)$ with $b(x, 0)=0$ for all $x$;

- $m_{0} \in C_{0}^{2+\gamma}\left(B_{1}\right)$ is an even function,

then the density function of (6) solves (9). Furthermore, $m$ has a representation via Fourier series, which goes to zero function as $t \rightarrow 0$.

\section{Proof of the main result}

In this section, we will prove Theorem 2. We outline the main proof of Theorem 2 in Section 3.1 based on some estimation results, whose proof will be provided in Section 3.2. We will use $K$ for a generic constant, and $K(\alpha, \beta)$ indicates its dependence on $\alpha$ and $\beta$. 


\subsection{Definition of the mapping $\mathcal{T}$ and the proof of Theorem 2}

We fix an arbitrary $T>0$ and $\gamma \in(0,1)$. We also define Banach spaces $\mathcal{B}$ and $\mathcal{R}$ given by

$$
\mathcal{B}=C^{1 / 2}\left((0, T) ; \mathbb{R}^{d}\right)
$$

and

$$
\mathcal{R}:=C^{2+\gamma, 1+\frac{\gamma}{2}}\left(B_{1} \times(0, T) ; \mathbb{R}\right) .
$$

For precise definitions of elliptic and parabolic Hölder spaces, we refer to Appendix A.1.

To proceed, we introduce an operator $\mathcal{T}: \mathcal{B} \mapsto \mathcal{B}$ through the composition $\mathcal{T}=\mathcal{T}_{1} \circ \mathcal{T}_{2}$, where $\mathcal{T}_{1}: \mathcal{B} \mapsto \mathcal{R}$ and $\mathcal{T}_{2}: \mathcal{R} \mapsto \mathcal{B}$ are defined as follows:

1. Define $\mathcal{T}_{1}: \beta \mapsto \mathcal{T}_{1}(\beta):=m$, where $m$ solves the following equation with a given process $\beta$,

$$
\begin{cases}\partial_{t} m=\frac{1}{2} \Delta m-\operatorname{div}_{x}\left(m b\left(x, \beta_{t}\right)\right), & \text { on } B_{1} \times(0, T) ; \\ m(x, 0)=m_{0}(x), & \text { on } \bar{B}_{1} ; \\ m(x, t)=0, & \text { on } \partial B_{1} \times(0, T) .\end{cases}
$$

2. Define $\mathcal{T}_{2}: m \mapsto \mathcal{T}_{2}(m)$, where

$$
\mathcal{T}_{2}(m)=\int_{B_{1}} x^{p} m_{t}(x) d x
$$

Proof: (of Theorem 2)

By Lemma $5, \mathcal{T}$ is a mapping from a Banach space $\mathcal{B}$ to itself. Furthermore, the mapping $\mathcal{T}: \mathcal{B} \mapsto \mathcal{B}$ has the following properties:

1. $\mathcal{T}$ is a continuous compact mapping by Lemma 6 ;

2. $\{x \in \mathcal{B}: x=\lambda \mathcal{T} x, \lambda \in[0,1]\}$ is bounded in $\mathcal{B}$ Lemma 7 .

By the Leray-Schauder's fixed point theorem (FPT), see Theorem 11.2 of [11], the mapping $\mathcal{T}$ has a fixed point in $\mathcal{B}$, i.e. there exists $\hat{\beta} \in \mathcal{B}$, such that

$$
\mathcal{T} \hat{\beta}=\hat{\beta} \text {. }
$$

If we set $\hat{m}=\mathcal{T}_{1} \hat{\beta}$, then the pair $(\hat{m}, \hat{\beta})$ solves FPK (9) by its definition of $\mathcal{T}=\mathcal{T}_{1} \circ \mathcal{T}_{2}$ in (16) -(17). Also due to Proposition 12, since $\hat{m}_{t}$ is the unique solution of (16), it is the density of the unique solution $\hat{X}_{t}$ of (7) with given $\beta=\hat{\beta}$. Together with the definition of $\mathcal{T}_{2}$ given by (17), the process $\hat{\beta}_{t}$ satisfies (8). Therefore, the pair $(\hat{X}, \hat{\beta})$ solves $(7)-(8)$, and this gives the solvability of (6) in the sense of Definition 1 .

\subsection{Estimates for the mapping $\mathcal{T}$}

In the proof of Theorem 2, we have used Lemma 5,6, 7 concerning the mapping $\mathcal{T}$, and we will present their proofs in this section separately. First, we shall verify that $\mathcal{T}$ is a well defined mapping. This includes 
1. the unique solvability of FPK (16);

2. Justification of the set $\mathcal{R}$ satisfying $\mathcal{T}_{1}(\mathcal{B}) \subset \mathcal{R} \subset \mathcal{T}_{2}^{-1}(\mathcal{B})$.

Lemma $3 \mathcal{T}_{1}: \mathcal{B} \mapsto \mathcal{R}$ is a well defined mapping with estimates

$$
\left|\mathcal{T}_{1}(\beta)\right|_{2+\gamma, 1+\gamma / 2} \leq K\left(|\beta|_{1 / 2}\right)\left|m_{0}\right|_{2+\gamma}
$$

ProOF: Rewrite the FPK (16) into non-divergence form

$$
\partial_{t} m=\frac{1}{2} \Delta m-b^{\beta} \circ \nabla m-m \operatorname{div}_{x}\left(b^{\beta}\right),
$$

where

$$
b^{\beta}(x, t)=b(x, \beta(t)) .
$$

Note that

- We have $b^{\beta} \in C^{1.0, \frac{1}{2}}\left(B_{1} \times(0, T)\right)$ by the application of Proposition 11 with (A1) and (14); Moreover,

$$
\left|-b^{\beta}\right|_{1.0,1 / 2}=|b|_{0}+\left[b^{\beta}\right]_{1.0,1 / 2} \leq K|b|_{1.0}\left(|\beta|_{1 / 2}+1\right) .
$$

- We also have $\operatorname{div}_{x}\left(b^{\beta}\right) \in C^{\gamma, \frac{\gamma}{2}}\left(B_{1} \times(0, T)\right)$. Indeed, one can write

$$
\operatorname{div}_{x}\left(b^{\beta}(x, t)\right)=\sum_{i=1}^{d} \partial_{x_{i}} b^{(i)}\left(x, \beta_{t}\right)
$$

and use Proposition 11 once again and the fact that $\partial_{x_{i}} b^{(i)} \in C^{\gamma}\left(B_{1}^{2}\right)$, which yields

$$
\left|\operatorname{div}_{x}\left(b^{\beta}\right)\right|_{\gamma, \gamma / 2} \leq \sum_{i=1}^{d}\left|\partial_{x_{i}} b^{(i)}\left(x, \beta_{t}\right)\right|_{0}+\sum_{i=1}^{d}\left[\partial_{x_{i}} b^{(i)}\left(x, \beta_{t}\right)\right]_{\gamma, \gamma / 2} \leq K|b|_{1+\gamma}\left(|\beta|_{1 / 2}+1\right) .
$$

Moreover, if we define $m_{0}^{T}(x, t)=m_{0}(x)$, then $m_{0}^{T} \in C^{1+\frac{\gamma}{2}, 2+\gamma}\left(B_{1} \times(0, T)\right)$. Therefore, by Theorem 10.3.3 of [2], there exists unique solution $m \in C^{2+\gamma, 1+\frac{\gamma}{2}}\left(B_{1} \times(0, T)\right)=\mathcal{R}$ for $(16)$.

If we set $\bar{m}(x, t)=e^{-\lambda t} m(x, t)$ with $\lambda=|b|_{1+\gamma}$, then $\operatorname{div}_{x}\left(b^{\beta}\right)+\lambda \leq 0$ and $\bar{m}$ solves

$$
\begin{cases}\partial_{t} \bar{m}=\frac{1}{2} \Delta \bar{m}-b^{\beta} \circ \nabla \bar{m}-\bar{m}\left(\operatorname{div}\left(b^{\beta}\right)+\lambda\right), & \text { on } B_{1} \times(0, T) \\ \bar{m}(x, 0)=m_{0}(x), & \text { on } \bar{B}_{1} ; \\ \bar{m}(x, t)=0, & \text { on } \partial B_{1} \times(0, T) .\end{cases}
$$

Now we can invoke the estimation from Proposition 13 to obtain

$$
|\bar{m}|_{2+\gamma, 1+\gamma / 2} \leq K\left(\left|b^{\beta}\right|_{\gamma, \gamma / 2},\left|\operatorname{div}_{x}\left(b^{\beta}\right)+\lambda\right|_{\gamma, \gamma / 2}\right)\left|m_{0}\right|_{2+\gamma}
$$

which is equivalent to

$$
|m|_{2+\gamma, 1+\gamma / 2} \leq K\left(|b|_{1+\gamma},|\beta|_{1 / 2}\right)\left|m_{0}\right|_{2+\gamma}
$$

Estimation of $\mathcal{T}_{2}$ directly follows from its definition. The Hölder space $C^{1.0}\left((0, T) ; \mathbb{R}^{d}\right)$ used below is indeed the space of Lipschitz continuous functions, see the remark on $C^{1.0}$ and $C^{1}$ in Appendix A.1. 
Lemma $4 \mathcal{T}_{2}: \mathcal{R} \mapsto C^{1.0}\left((0, T) ; \mathbb{R}^{d}\right)$ is well defined with an estimate

$$
\left|\mathcal{T}_{2}(m)\right|_{1.0} \leq K|m|_{2+\gamma, 1+\frac{\gamma}{2}} .
$$

ProOf: To prove Lipschitz continuity of the process $\mathcal{T}_{2}(m)$, we shall show

$$
\left|\mathcal{T}_{2}(m)\left(t_{1}\right)-\mathcal{T}_{2}(m)\left(t_{2}\right)\right| \leq K\left|t_{1}-t_{2}\right|, \quad \forall 0 \leq t_{1}<t_{2} \leq T .
$$

This follows from the following estimate for the mapping $\mathcal{T}_{2}$ of $m \in \mathcal{R}$ and $0<t_{1} \leq t_{2}<T$ :

$$
\begin{aligned}
\left|\mathcal{T}_{2}(m)\left(t_{1}\right)-\mathcal{T}_{2}(m)\left(t_{2}\right)\right| & \leq \int_{B_{1}}|x|^{p}\left|m\left(x, t_{1}\right)-m\left(x, t_{2}\right)\right| d x \\
& =\int_{B_{1}}|x|^{p} \cdot \int_{t_{1}}^{t_{2}}\left|\partial_{t} m\right|(x, s) d s d x \\
& \leq \int_{B_{1}}|x|^{p} d x|m|_{2+\gamma, 1+\frac{\gamma}{2}}\left|t_{2}-t_{1}\right| .
\end{aligned}
$$

Now we can have an estimation of $\mathcal{T}$ as a well-defined mapping.

Lemma $5 \mathcal{T}: \mathcal{B} \mapsto C^{1.0}\left((0, T) ; \mathbb{R}^{d}\right) \subset \mathcal{B}$ is well defined with

$$
|\mathcal{T}(\beta)|_{1.0} \leq K\left(|\beta|_{\frac{1}{2}}\right)\left|m_{0}\right|_{2+\gamma} .
$$

ProOF: It is a consequence of Lemma 3 and 4.

So far, we established that the operator $\mathcal{T}$ is well defined from its domain $\mathcal{B}$ to itself. Next, we will prove key facts for the proof of the fixed point theorem: the continuity and the compactness of the operator $\mathcal{T}$. For this purpose, we shall briefly recall the following imbedding properties on Hölder spaces. Consider two Hölder spaces $C^{\gamma}$ and $C^{\lambda}$ for $\gamma>\lambda>0$. Then, $C^{\gamma} \subset C^{\lambda}$ holds and any bounded subset of $C^{\gamma}$ is a compact subset of $C^{\lambda}$. Furthermore, if (1) $C^{\gamma} \ni \alpha_{n} \rightarrow \alpha$ pointwisely; and (2) $\left|\alpha_{n}\right|_{\gamma}<K$ for any $n \in \mathbb{N}$, then $\alpha_{n} \rightarrow \alpha$ in $C^{\lambda}$, i.e. $\left|\alpha_{n}-\alpha\right|_{\lambda} \rightarrow 0$ as $n \rightarrow \infty$. However, $\alpha_{n} \rightarrow \alpha$ in $C^{\gamma}$ (i.e. $\left|\alpha_{n}-\alpha\right|_{\gamma} \rightarrow 0$ ) may not be true.

Lemma $6 \mathcal{T}$ is continuous compact in $\mathcal{B}$.

Proof: Lemma 5 implies that any sequence $\left\{\beta^{n}\right\}$ bounded in $\mathcal{B}=C^{1 / 2}\left((0, T) ; \mathbb{R}^{d}\right)$ maps to a sequence $\left\{\mathcal{T}\left(\beta^{n}\right)\right\}$ bounded in $C^{1.0}\left((0, T) ; \mathbb{R}^{d}\right)$, which is precompact in $C^{1 / 2}\left((0, T) ; \mathbb{R}^{d}\right)$. Thus, $\mathcal{T}$ is compact.

In this below, we will establish the continuity of the mapping $\mathcal{T}$. If $\beta^{n} \rightarrow \beta^{\infty}$ in $\mathcal{B}$, we denote, for simplicity

$$
m^{n}=\mathcal{T}_{1}\left(\beta^{n}\right), \alpha^{n}=\mathcal{T}_{2}\left(m^{n}\right), \forall n \in \mathbb{N} \cup\{\infty\} .
$$

Our objective is to show $\alpha^{n} \rightarrow \alpha^{\infty}$ in $\mathcal{B}$. Since $\left\{m^{n}: n \in \mathbb{N}\right\}$ is bounded in $C^{2+\gamma, 1+\frac{\gamma}{2}}\left(B_{1} \times(0, T) ; \mathbb{R}\right)$ by Lemma 3, the pointwise convergence of $m^{n}$ as $n \rightarrow \infty$ holds from stability of the viscosity solution, i.e.

$$
m^{n}(x, t) \rightarrow m^{\infty}(x, t), \forall(x, t) \in B_{1} \times(0, T) .
$$

Since $\left\{m^{n}: n \in \mathbb{N}\right\}$ is bounded in $C^{2+\gamma, 1+\frac{\gamma}{2}}\left(B_{1} \times(0, T) ; \mathbb{R}\right)$ by Lemma 3 , one can use bounded convergence theorem to obtain,

$$
\alpha^{n}(t)-\alpha^{\infty}(t)=\int_{B_{1}} x^{p} \cdot\left(m^{n}(x, t)-m^{\infty}(x, t)\right) d x \rightarrow 0, \quad \forall t \in(0, T) .
$$


Thus, $\alpha^{n}$ converges $\alpha^{\infty}$ pointwisely. But we know $\left\{\alpha^{n}: n \in \mathbb{N}\right\}$ are bounded in $C^{1.0}\left((0, T) ; \mathbb{R}^{d}\right)$ by Lemma 5 . Pointwise convergence and boundedness in $C^{1.0}\left((0, T) ; \mathbb{R}^{d}\right)$ implies convergence in $C^{1 / 2}\left((0, T) ; \mathbb{R}^{d}\right)$, i.e. $\alpha^{n} \rightarrow \alpha^{\infty}$ in $\mathcal{B}$.

The following is the last piece required to complete the FPT.

Lemma 7 The set $\{\beta: \beta=\lambda \mathcal{T} \beta, \lambda \in[0,1]\}$ is bounded in $\mathcal{B}$.

Proof: We shall show that, there exists $M>0$, s.t. if $\beta$ solves (18) below for some $\lambda \in[0,1]$, then $|\beta|_{C^{1 / 2}\left((0, T) ; \mathbb{R}^{d}\right)}<M$.

$$
\begin{cases}\partial_{t} m=\frac{1}{2} \Delta m-\operatorname{div}_{x}\left(b\left(x, \beta_{t}\right) m\right), & B_{1} \times(0, T) \\ m(x, 0)=m_{0}(x), & x \in \bar{B}_{1} \\ m(x, t)=0, & \partial B_{1} \times(0, T) \\ \beta_{t}=\lambda \int_{B_{1}} x^{p} m(x, t) d x & t \in(0, T) .\end{cases}
$$

If $\lambda=0$, then $\beta=0$, then the conclusion trivially holds. If $\lambda \in(0,1]$, we first have

$$
|\beta|_{0} \leq \sup _{t \in(0, T)} \lambda \int_{B_{1}}|x|^{p} m(x, t) d x \leq \sup _{t \in(0, T)} \int_{B_{1}} m(x, t) d x \leq 1 .
$$

So, it's enough to show the boundedness of $[\beta]_{1 / 2}$. To proceed, we write the stochastic representation by Proposition 12 as follows.

$$
\left\{\begin{array}{l}
d X_{t}=b\left(X_{t}, \beta_{t}\right) d t+d W_{t}, \\
\tau=\inf \left\{t>0: X_{t} \notin B_{1}\right\} \\
\beta(t)=\lambda \mathbb{E}^{m_{0}}\left[\left|X_{t}\right|^{p} I_{[0, \tau)}(t)\right] .
\end{array}\right.
$$

Without loss of generality, we set $0<t_{1}<t_{2}<T$. Then, we have

$$
\begin{aligned}
\left|\beta\left(t_{1}\right)-\beta\left(t_{2}\right)\right|^{2} & =\lambda^{2}\left|\mathbb{E}^{m_{0}}\left[X_{t_{1}} I_{[0, \tau)}\left(t_{1}\right)\right]-\mathbb{E}^{m_{0}}\left[X_{t_{2}} I_{[0, \tau)}\left(t_{2}\right)\right]\right|^{2} \\
& \leq 2 \lambda^{2}\left|\mathbb{E}^{m_{0}}\left[X_{t_{1}} I_{\left[t_{1}, t_{2}\right]}(\tau)\right]\right|^{2}+2 \lambda^{2}\left|\mathbb{E}^{m_{0}}\left[\left(X_{t_{1}}-X_{t_{2}}\right) I_{\left[t_{2}, T\right]}(\tau)\right]\right|^{2} \\
& \leq 2 \lambda^{2}\left|\mathbb{E}^{m_{0}}\left[I_{\left[t_{1}, t_{2}\right]}(\tau)\right]\right|^{2}+2 \lambda^{2} \mathbb{E}^{m_{0}}\left[\left|X_{t_{1}}-X_{t_{2}}\right|^{2}\right] \\
& \leq \lambda^{2} K\left(|b|_{0}\right)\left|t_{1}-t_{2}\right|, \quad \text { by (D9) of }[10] .
\end{aligned}
$$

Therefore, we have

$$
[\beta]_{1 / 2} \leq \lambda K^{1 / 2}\left(b_{0}\right) .
$$

Thus, if we choose $M=1+\lambda K^{1 / 2}\left(\left|b_{0}\right|\right)$, we shall have

$$
|\beta|_{1 / 2}=|\beta|_{0}+[\beta]_{1 / 2} \leq 1+\lambda K^{1 / 2}\left(\left|b_{0}\right|\right)=M .
$$

\section{Summary}

In this note, we showed that under assumption (A1) on the drift function and the initial density, the killed McKean-Vlasov process (6) solves FPK (9). We observe that uniqueness of the solution 
to the FPK (9) has not been established in this paper and this will be the subject of future work. If the uniqueness were true, as an application, one can approximate the population $p$ th mean(5) of large system in a bounded region by its associated FPK. It may also be interesting to consider the estimates of the population $p$ th moment normalized by the number of survivals, which is closely related to $Y^{N}$ given by (5).

\section{A Appendix}

\section{A.1 Hölder Space}

For convenience, we introduce the notion of elliptic Hölder space $C^{k+\gamma}(\mathcal{D} ; \mathcal{R})$ and parabolic Hölder space $C^{\frac{\gamma}{2}, \gamma}(\mathcal{D} \times(0, T) ; \mathcal{R})$ from [16]. In this paper, the domain $\mathcal{D}$ may be $B_{1}, B_{1}^{2}$ or $(0, T)$; and the range $\mathcal{R}$ may be $\mathbb{R}$ or $\mathbb{R}^{d}$. If the range $\mathcal{R}$ is $\mathbb{R}$, then $\mathcal{R}$ may be abbreviated, for instance, $C^{\gamma}(\mathcal{D})$ means $C^{\gamma}(\mathcal{D} ; \mathbb{R})$. By uniform continuity, we shall treat $C^{\gamma}(\mathcal{D})$ as the same as $C^{\gamma}(\overline{\mathcal{D}})$ by natural bijective isometry.

\section{A.1.1 Elliptic Hölder Space}

Let $\mathcal{D}$ be a domain in $\mathbb{R}^{d}$ and $\mathcal{R}$ be a range in $\mathbb{R}^{d_{1}}$. For $u: \mathcal{D} \mapsto \mathcal{R}$, we define a uniform norm by $|u|_{0}=\sup _{\mathcal{D}}|u|$, and we denote by $\partial_{x_{i}}^{\alpha_{i}} u$ the $\alpha_{i}$-th order partial derivative in the variable $x_{i}$, if it exists. For multiindex $\alpha=\left(\alpha_{i}: i=1, \ldots d\right)$, we use $D^{\alpha} u=\partial_{x_{1}}^{\alpha_{1}} \cdots \partial_{x_{d}}^{\alpha_{d}} u$.

For $k \in \mathbb{N} \cup\{0\}$, we denote by $C_{\text {loc }}^{k}(\mathcal{D}, \mathcal{R})$ the set of all functions $u: \mathcal{D} \mapsto \mathcal{R}$ whose derivatives $D^{\alpha} u$ for $|\alpha| \leq k$ are continuous in $\mathcal{D}$. One can define a norm in $C_{l o c}^{k}(\mathcal{D}, \mathcal{R})$ by

$$
|u|_{k}=\sum_{i=0}^{k} \max _{|\alpha|=i}\left|D^{\alpha} u\right|_{0} .
$$

Then the functions $u$ having finite norm consists of Banach space, and we refer it to $C^{k}(\mathcal{D}, \mathcal{R})$. For instance, $u=e^{x}: \mathbb{R} \mapsto \mathbb{R}$ belongs to $C_{\text {loc }}^{k}(\mathbb{R}, \mathbb{R})$ but not $C^{k}(\mathbb{R}, \mathbb{R})$.

For $\gamma \in(0,1]$, we can also define a Hölder seminorm for a function $u \in C(\mathcal{D}, \mathbb{R})$ by,

$$
[u]_{\gamma}=\sup _{x, y \in \mathcal{D}, x \neq y} \frac{|u(x)-u(y)|}{|x-y|^{\gamma}} .
$$

Definition 8 For a decimal number $\gamma \in(0,1]$ and an integer $k \in \mathbb{N} \cup\{0\}$, Hölder space $C^{k+\gamma}(\mathcal{D}, \mathcal{R})$ is the Banach space of all functions $u \in C^{k}(\mathcal{D}, \mathcal{R})$ for which the norm

$$
|u|_{k+\gamma}=|u|_{k}+\max _{|\alpha|=k}\left[D^{\alpha} u\right]_{\gamma}
$$

is finite.

In the above, we emphasize that $\gamma$ is a decimal number (writing with decimal point) and $k$ is an integer to avoid the following ambiguity. Note that $C^{1.0}(\mathcal{D}, \mathcal{R})$ is 1-Hölder space (or Lipschitz continuous space) with a finite norm w.r.t.

$$
|u|_{1.0}=|u|_{0}+\sup _{x, y \in \mathcal{D}, x \neq y} \frac{|u(x)-u(y)|}{|x-y|^{\gamma}}
$$


while $C^{1}(\mathcal{D}, \mathcal{R})$ is a continuous differentiable function space with a finite norm w.r.t.

$$
|u|_{1}=|u|_{0}+\max _{i=1}^{d}\left|\partial_{x_{i}} u\right|_{0} .
$$

For instance, $f(x)=|x|$ is in $C^{1.0}([-1,1]) \backslash C^{1}([-1,1])$ with its norm

$$
|f|_{1.0}=|f|_{0}+[f]_{1.0}=2 .
$$

Another example is that $g(x)=x^{2} \operatorname{sgn}(x)$ is in $C^{2.0}\left([-1,1] \backslash C^{2}([-1,1])\right.$ with

$$
|g|_{2.0}=|g|_{0}+\left|g^{\prime}\right|_{0}+\left[g^{\prime}\right]_{1.0}=5 .
$$

In general, $C^{k+1}(\mathcal{D}, \mathcal{R})$ is a proper subset of $C^{k+1.0}(\mathcal{D}, \mathcal{R})$.

Next, we use the extension of $u: \mathcal{D} \mapsto \mathcal{R}$ with $\tilde{u}(x)=u(x) I_{\mathcal{D}}(x): \mathbb{R}^{d} \mapsto \mathbb{R}^{d_{1}}$ by taking values the same as $u$ in $\mathcal{D}$ otherwise zero.

Definition 9 Let $\mathcal{D}$ be a bounded set in $\mathbb{R}^{d}$. The space $C_{0}^{k+\gamma}(\mathcal{D}, \mathcal{R})$ is defined by

$$
C_{0}^{k+\gamma}(\mathcal{D}, \mathcal{R})=\left\{u \in C^{k+\gamma}(\mathcal{D}, \mathcal{R}): u(x) I_{\mathcal{D}}(x) \in C^{k+\gamma}\left(\mathbb{R}^{d}, \mathcal{R}\right)\right\} .
$$

\section{A.1.2 Parabolic Hölder Space}

Let $\mathcal{D}$ be a domain in $\mathbb{R}^{d}, \mathcal{Q}=\mathcal{D} \times(0, T)$ be the parabolic domain in $\mathbb{R}^{d+1}$ for some $T>0$, and $\mathcal{R}$ be a range in $\mathbb{R}^{d_{1}}$. We are going to define norms for $u: \mathcal{Q} \mapsto \mathcal{R}$ in this below.

First, we define parabolic metric on $\mathbb{R}^{d+1}$ : for any $z_{1}=\left(x_{1}, t_{1}\right), z_{2}=\left(x_{2}, t_{2}\right) \in \mathbb{R}^{d+1}$

$$
\rho\left(z_{1}, z_{2}\right)=\left|x_{1}-x_{2}\right|+\left|t_{1}-t_{2}\right|^{1 / 2} .
$$

Then, we set the parabolic Hölder seminorm for $u \in C(\mathcal{Q})$ by, $\gamma \in(0,1)$

$$
[u]_{\gamma, \gamma / 2}=\sup _{z_{1}, z_{2} \in \mathcal{Q}, z_{1} \neq z_{2}} \frac{\left|u\left(z_{1}\right)-u\left(z_{2}\right)\right|}{\rho^{\gamma}\left(z_{1}, z_{2}\right)} .
$$

Definition 10 For $\gamma \in(0,1]$ and $k \in \mathbb{N} \cup\{0\}$, the parabolic Hölder space $C^{2 k+\gamma, k+\gamma / 2}(\mathcal{Q}, \mathcal{R})$ is the Banach space of all functions $u \in C(\mathcal{Q}, \mathcal{R})$ for which the norm

$$
|u|_{2 k+\gamma, k+\gamma / 2}=|u|_{0}+\sum_{i=1}^{k}\left|D_{t}^{i} u\right|_{0}+\sum_{i=1}^{2 k} \max _{|\alpha|=i}\left|D_{x}^{\alpha} u\right|_{0}+\max _{|\alpha|=2 k}\left[D_{t}^{k} D_{x}^{\alpha} u\right]_{\gamma, \gamma / 2} .
$$

is finite.

In this text, we only use $C^{2 k+\gamma, k+\gamma / 2}(\mathcal{Q}, \mathcal{R})$ for $k=0,1$. We also need the following elementary fact between elliptic Hölder and parabolic Hölder spaces.

Proposition 11 If $f \in C^{\delta}\left((0, T) ; B_{1}\right)$ and $g \in C^{\gamma}\left(B_{1}^{2}\right)$ be two functions for some constants $\delta \in$ $(0,1 / 2]$ and $\gamma \in(0,1]$, then $h(x, t)=g(x, f(t))$ belongs to $C^{2 \delta \gamma, \delta \gamma}\left(B_{1} \times(0, T)\right)$ with

$$
[h]_{2 \delta \gamma, \delta \gamma} \leq K[g]_{\gamma}\left([f]_{\delta}+1\right) .
$$

Proof: The result follows from the following inequalities:

$$
\left|h\left(x_{1}, t_{1}\right)-h\left(x_{2}, t_{2}\right)\right|=\left|g\left(x_{1}, f\left(t_{1}\right)\right)-g\left(x_{2}, f\left(t_{2}\right)\right)\right| \leq[g]_{\gamma}\left(\left|f\left(t_{1}\right)-f\left(t_{2}\right)\right|^{2}+\left|x_{1}-x_{2}\right|^{2}\right)
$$

Since $\left|f\left(t_{1}\right)-f\left(t_{2}\right)\right| \leq[f]_{\delta}\left|t_{1}-t_{2}\right|^{\delta},\left|t_{1}-t_{2}\right|+\left|x_{1}-x_{2}\right| \leq 3$, and $2 \delta \leq 1$, we conclude that

$$
\left|h\left(x_{1}, t_{1}\right)-h\left(x_{2}, t_{2}\right)\right| \leq K[g]_{\gamma}\left([f]_{\delta}+1\right)\left(\left|t_{1}-t_{2}\right|^{1 / 2}+\left|x_{1}-x_{2}\right|\right)^{2 \delta \gamma} .
$$




\section{A.2 Relations between PDEs and SDE without mean field term}

We consider the density of a killed process on the unit ball $B_{1}$ given by

$$
d X_{t}=\left(\bar{b}\left(X_{t}, t\right) d t+d W_{t}\right) I_{B_{1}}\left(X_{t}\right), X_{0} \sim m_{0}
$$

for a function $\bar{b} \in C^{2+\delta, 1+\frac{\delta}{2}}\left(B_{1} \times(0, T)\right)$. One can define a semigroup $\left\{P_{s, t}: 0 \leq s \leq t\right\}$ on a Banach space $C_{0}=C_{0}\left(B_{1}\right)$ by

It can be checked that

$$
P_{s, t} f(x)=\mathbb{E}^{x, s}\left[f\left(X_{t}\right)\right] .
$$

- $P_{t, t}=I$

- $P_{s, t} P_{t, r}=P_{s, r}$ for $0 \leq s \leq t \leq r$;

Recall that the generator

$$
L_{t} f(x)=\lim _{h} \frac{P_{t, t+h}-I}{h} f(x) .
$$

In this case, the generator can be written explicitly as

$$
L_{t} f(x)=\bar{b} \circ \nabla f+\frac{1}{2} \Delta f
$$

and the domain $\mathcal{D}\left(L_{t}\right)$ of the generator includes the smooth test function set $C_{0}^{\infty}\left(B_{1}\right)$. Moreover, the adjoint operator of $L_{t}$ is given by

$$
L_{t}^{*} f(x)=-\operatorname{div}_{x}(\bar{b} f)+\frac{1}{2} \Delta f .
$$

Formally, if we denote the density of $X_{t}$ on $B_{1}$ by $m(t, \cdot)$, i.e.

$$
P_{0, t} f(x)=\left(m_{t}, f\right), \forall f \in C_{0} .
$$

It is also noted that, $X_{t}$ is a submarkovian on $B_{1}$, since $\int_{B_{1}} m_{t}(x) d x=1-\mathbb{P}\left(X_{t} \in \partial B_{1}\right) \leq 1$. One can carry out

$$
\frac{d}{d t} P_{s, t} f(x)=\lim _{h} \frac{P_{s, t+h}-P_{s, t}}{h} f(x)=P_{s, t} L_{t} f(x), \forall f \in C_{0}^{\infty} .
$$

Taking $s=0$, it becomes

$$
\frac{d}{d t}\left(m_{t}, f\right)=\left(m_{t}, L_{t} f\right), \forall f \in C_{0}^{\infty}
$$

which implies the Kolmogorov forward equation with appropriate initial-boundary conditions:

$$
\begin{cases}\partial_{t} m=L_{t}^{*} m, & B_{1} \times(0, T), \\ m(x, 0)=m_{0}(x), & x \in \bar{B}_{1}, \\ m(x, t)=0, & \partial B_{1} \times(0, T) .\end{cases}
$$

Next, if we denote $u(x, t)=P_{t, T} g(x)$ for some fixed $g \in C_{0}$, and $u \in C^{1,2}$ for some fixed $T$, then we can write

$$
\frac{d}{d t} P_{t, r} g(x)=\lim _{h} \frac{P_{t+h, r}-P_{t, r}}{h} g(x)=\lim _{h} \frac{I-P_{t, t+h}}{h} P_{t+h, r} g(x)=-L_{t} P_{t, r} g(x) .
$$

This implies, by taking $r=T$, the Kolmogorov backward equation with some terminal-boundary conditions:

$$
\begin{cases}\partial_{t} u+L_{t} u=0, & B_{1} \times(0, T), \\ u(x, T)=g(x), & x \in \bar{B}_{1}, \\ u(x, t)=0, & \partial B_{1} \times(0, T) .\end{cases}
$$




\section{Proposition 12}

Assume $\bar{b} \in C^{2+\delta, 1+\frac{\delta}{2}}\left((0, \infty) \times B_{1}\right), m_{0}, g \in C_{0}^{\delta}\left(B_{1}\right)$. Then,

1. The density $m(x, t)$ of $X$ of (19) on the open set $B_{1}$ is the unique solution of (20) in $C^{2+\delta, 1+\delta / 2}$;

2. $u(x, t)=P_{t, T} g(x)$ is the unique solution of $(21)$ in $C^{2+\delta, 1+\delta / 2}$.

Proof: One can first write down non-divergence form of (20). Then, the uniqueness of the solution and its regularity result of (20) and (21) directly follows from Theorem 10.3.3 of [2]. The relation of (20) and transition density of the submarkovian process $X$ is referred to Section 4.1 of [18]. The stochastic representation of (21) to the function $P_{t, T} g(x)$ is referred to Section 40.2 of [1].

We need the following estimate in the sequel. Consider

$$
\begin{cases}\partial_{t} u=L_{t} u+c u, & B_{1} \times(0, T), \\ u(x, 0)=g(x), & x \in \bar{B}_{1}, \\ u(x, t)=0, & \partial B_{1} \times(0, T) .\end{cases}
$$

\section{Proposition 13}

If $b, c \in C^{\delta, \delta / 2}, g \in C_{0}^{2+\delta}$, and $c \leq 0$, then (22) is uniquely solvable satisfying

$$
|u|_{2+\delta, 1+\delta / 2} \leq K\left(|b|_{\delta, \delta / 2},|c|_{\delta, \delta / 2}\right)|g|_{2+\delta} .
$$

Proof: Unique solvability is implied by Theorem 10.3 .3 of [16] and the estimate is given by Theorem 10.2.2 of [16].

\section{References}

[1] Richard F. Bass. Stochastic processes, volume 33 of Cambridge Series in Statistical and Probabilistic Mathematics. Cambridge University Press, Cambridge, 2011. 13

[2] A. Bensoussan, J. Frehse, and P. Yam. Mean field games and mean field type control theory. Springer, New York, 2013. 1

[3] Luciano Campi and Markus Fischer. $n$-player games and mean-field games with absorption. Ann. Appl. Probab., 28(4):2188-2242, 08 2018. 2

[4] P Cardaliaguet. Notes on mean field games. https://www.ceremade.dauphine.fr/ cardaliaguet/MFG20130420.pdf, 2013. 1, 2

[5] R. Carmona, J. Fouque, and L. Sun. Mean field games and systemic risk. Commun. Math. Sci, 13(4), 2015. 2

[6] Kai Lai Chung and John B. Walsh. Markov processes, Brownian motion, and time symmetry, volume 249 of Grundlehren der Mathematischen Wissenschaften [Fundamental Principles of Mathematical Sciences]. Springer, New York, second edition, 2005. 3

[7] Jakša Cvitanić, Jin Ma, and Jianfeng Zhang. The Law of Large Numbers for self-exciting correlated defaults. Stochastic Process. Appl., 122(8):2781-2810, 2012. 2

[8] William Feller. An introduction to probability theory and its applications. Vol. II. Second edition. John Wiley \& Sons, Inc., New York-London-Sydney, 1971. 5 
[9] D. Firoozi, A. Pakniyat, and P. E. Caines. A mean field game - hybrid systems approach to optimal execution problems in finance with stopping times. In: Proceedings of the 56th IEEE $C D C, 2017.2$

[10] W. H. Fleming and H. M. Soner. Controlled Markov processes and viscosity solutions, volume 25 of Stochastic Modelling and Applied Probability. Springer, New York, second edition, 2006. 9

[11] D. Gilbarg and N. S. Trudinger. Elliptic partial differential equations of second order. Classics in Mathematics. Springer-Verlag, Berlin, 2001. Reprint of the 1998 edition. 6

[12] Diogo A. Gomes, Edgard A. Pimentel, and Vardan Voskanyan. Regularity theory for mean-field game systems. SpringerBriefs in Mathematics. Springer, [Cham], 2016. 1

[13] M. Huang, P. E. Caines, and R. P. Malhame. Individual and mass behavior in large population stochastic wireless power control problems: centralized and nash equilibrium solutions. In: Proceedings of the 42nd IEEE CDC, pages 98-103, 2003. 1

[14] M. Huang, P. E. Caines, and R. P. Malhame. Large population stochastic dynamic games: closed- loop mckean-vlasov systems and the nash certainty equivalence principle. Commun Inf Syst, 6(3):221-251, 2006. 1

[15] M. Huang, P. E. Caines, and R. P. Malhame. Large-population cost-coupled lqg problems with non-uniform agents: individual-mass behavior and decentralized epsilon-nash equilibria. IEEE Trans Automatic Control, 52:1560-1571, 2007. 1

[16] N. V. Krylov. Lectures on elliptic and parabolic equations in Hölder spaces, volume 12 of Graduate Studies in Mathematics. American Mathematical Society, Providence, RI, 1996. 4, 10,13

[17] J-M Lasry and P-L Lions. Mean field games. Japan J. Math, 2:229-260, 2007. 1

[18] Grigorios A. Pavliotis. Stochastic processes and applications, volume 60 of Texts in Applied Mathematics. Springer, New York, 2014. Diffusion processes, the Fokker-Planck and Langevin equations. 13

[19] L. C. G. Rogers and David Williams. Diffusions, Markov processes, and martingales. Cambridge Mathematical Library. Cambridge University Press, Cambridge, 2000. Foundations, Reprint of the second (1994) edition. 1

[20] Daniel W. Stroock. Partial differential equations for probabilists, volume 112 of Cambridge Studies in Advanced Mathematics. Cambridge University Press, Cambridge, 2008. 5 